\title{
Leader-Follower Framework for Control of Energy Services
}

\author{
Ali Keyhani, Fellow, IEEE
}

\begin{abstract}
This paper presents a framework for leader-follower control of energy services. In this framework, the main emphasis is to provide market players with proper economic signals in order for the market to function efficiently in light of market players' profit motives and system reliability and security requirements. The problem formulation and the data needed for the implementation of the proposed framework are also presented.
\end{abstract}

Index Terms-Energy services market, leader-follower optimization, predictive market monitoring, reliability and competitiveness of operation.

\section{INTRODUCTION}

$\mathbf{T}$ HE problems of the reliability of the electric energy market have made it imperative to recognize that market forces motivated by profit cannot guarantee a secure and reliable energy supply because of the inherent characteristics of electric energy production, transmission and distribution networks. No other systems, such as transportation, gas distribution, airline, and trucking are subjected to high prices due to outages from the lack of maintenance of one of its components.

Many researchers have concluded that electric energy production is a product and have studied problems related to free market operation of electric energy systems. [1]-[17] Others, [18]-[20] have argued that electricity is a service and not a product with unique operational requirements. Casazza [18] points out that the operational time constant for electric power is zero, and the planning time constant for electric energy services is two to 10 years. Casazza presents an excellent analysis of how free market planning and operation of electric services have created many new problems in security and reliability of electric energy services in a paper entitled " Electricity Choice: Pick your Poison.” Power Systems engineers have recognized these issues and have studied these problems. Alvarado [6] has shown the dynamic coupling between the power market and power systems. Billington [14] and Rau [7] studied the problems of capacity reserve assessment and assignment of capability obligation. Rau and Billington's work addresses the reliability issues that are created under the new free market operation.

To address the problems of reliability and security, this paper presents the restructuring of electric energy services where the market players' profit motives will mesh with system security

Manuscript received July 5, 2001; revised February 20, 2002. This work was supported in part by the National Science Foundation under Grant ECS-0118080.

A. Keyhani is with the Department of Electrical Engineering, The Ohio State University, Columbus, OH 43210 USA (e-mail: Keyhani.1@ osu.edu).

Digital Object Identifier 10.1109/TPWRS.2003.810985 and reliability. The approach is based on the Stackelberg market design strategy developed in the 1970s [19]-[22].

\section{A. Power Systems Planned Operation}

Each power plant has a different production cost that depends on the unit's size, its fuel type, location, and age. As energy demand increases during daily operation, the clearing price goes up until it matches the production cost of the most expensive supply. If there is a supply shortage (true or contrived), this process could raise the price enormously, as inelastic demand will have to settle for any price bid by suppliers. During the 2000 power crisis in California, price caps were implemented by the independent system operator (ISO) to mitigate market power concerns. Predictably, energy prices immediately followed the price caps, creating a form of simple leader-follower phenomenon without proper incentive functions. The imposition of blackouts by the California ISO indirectly created a form of retail price elasticity. It can be shown that even under normal system conditions, the market can be manipulated to raise prices. For example, a market player with several plants in one state could bid the output of one of its plants at a very high price to see if it could drive up the overall price at auction.

The following requirements are known to energy system planners

a) Long term reliability and adequacy of energy systems require that an adequate capacity of electric energy be installed in the face of growing energy demands with the right mix of fuel supply to ensure that energy prices will not be subjected to very high volatility due to the shortage of a particular fuel. The generators must have the correct dynamic responses if the system is to remain stable in the event of an outage.

b) A nuclear unit and once-through steam unit cannot participate in regulating the time varying loads. The mix of generating systems with the right dynamic response is essential for the system to operate efficiently, securely, and reliably.

c) Coordination between transmission systems, generating systems, and load centers is a must if energy is to reach the load centers.

d) It takes a minimum of two years to build a reasonable size generating system at the right location in the network. One cannot simply build a unit if it is not properly coordinated within the transmission system. Furthermore, if a unit is not properly planned, it could block another unit planned by another market player. 
The above requirements, among many others, indicate that market forces alone cannot ensure adequate security and reliability of power systems.

The three fundamental problems of controlling energy services are: (1) determination of expected future demands; (2) calculation of a time-varying price of energy; and (3) the interrelationship between market players and market and system operators (e.g., the structure of the market). The first and second problems require the development of dynamic models based on historical data and all observable inputs to the process. In this paper, the leader-follower game theory is used to formulate a solution for control of energy services based on the security and reliability requirements of the system. The main function of market coordination is assigned to a market monitoring board (MMB) that has the responsibility to ensure market competition. Based on the leader-follower optimization method, the MMB will plan the operation of the system. The proposed leader-follower algorithm will enable the MMB to monitor the system by designing a set of market competitive functions (MCF). The framework of this market is depicted in Fig. 1. The MMB is responsible for the long-term planning and operation of the system. The MMB will also monitor all demand and supply bids for both forward market and daily operation of the system to ensure compliance with the MCF functions. As shown in Fig. 1, the bids that are in compliance with the MCF functions are sent to the energy market. The MCF functions are expressed as a system of equations and are calculated based on the amount of power that can be injected into each bus, plus a rational price based on the unit type. The market players will work with the MCF functions to develop their quantity and price bids for energy services.

In the market structure proposed in Fig. 1, the state will determine the spinning reserve energy requirements under the MMB control in order to ensure that required security and reliability of the system are attained. The MMB will estimate the cost of the load being served, act as a player, and select a load response program to supply the spinning reserve requirements. In Section II, the methodology for computation of the value of the load loss will be described and will be followed by the formulation of the leader-follower algorithms.

\section{Motivation AND PROBLEM Formulation}

In the proposed method, the MMB would be an independent, technically and politically enabled entity that, on a proactive and predictive fashion, determines market competitiveness, and calculates and disseminates the MCFs to market players. The MCF's set boundaries for the behavior of market players such that market power abuses do not take place and system reliability and security concerns are addressed.

In order to determine the MCFs, the MMB must first determine the system reliability index (SRI) based on the transmission reliability index (TRI) and generation reliability index (GRI).

The MMB can calculate the value of the load loss and spinning reserve as presented later in this paper. To ensure system security, the required power could be provided by the players

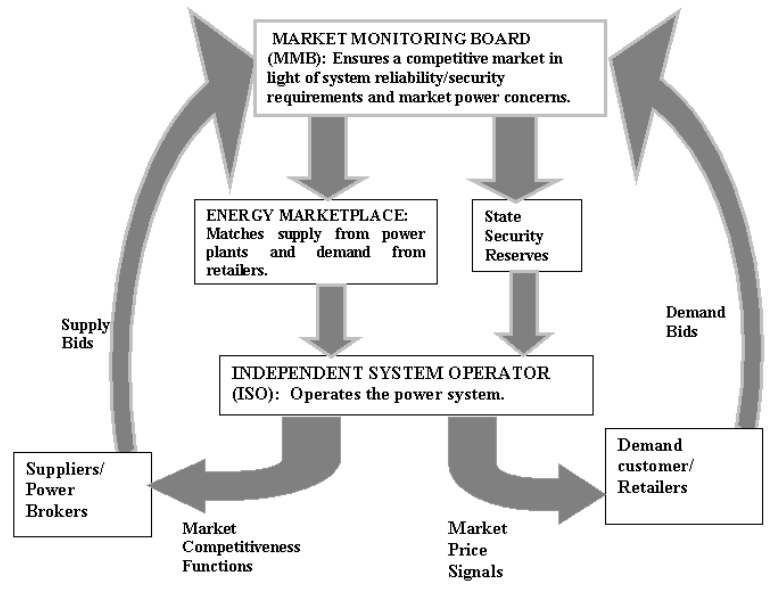

Fig. 1. Leader-follower market framework for control of energy services.

at a rational price to satisfy the spinning reserve requirements, or if the offering price of the players is not reasonable, the state reserves can be scheduled, or the load response programs activated to reduce demand.

\section{A. Computation of Value of Loss of Load (VOLL)}

Since the nature of consumers' energy consumption, major market participants' strategic behavior and power system reliability indices are stochastic processes; the energy price model will be stochastic as well [23]-[27]. In our analysis, the price model of electricity is based on the consumers' hourly consumption, day-ahead/real-time energy prices, generation and transmission outage schedules (evaluation of the power system reliability indices), spinning reserve requirements, demand elasticity and predicted strategic behavior of market participants. The obtained dynamic price model is used to evaluate the consumers' value of lost load (VOLL) for optimal price tariff design and pricing of spinning reserve. The following covariance model will be used as a candidate for price prediction:

$$
\begin{aligned}
\hat{p}_{k+1}= & E\left(p_{k+1} \mid k\right) \\
= & p_{k}+\sum_{j=1}^{N}\left\{A_{j} \operatorname{cov}\left(p_{k}, x_{j}\right) \operatorname{var}\left(x_{j}\right)^{-1}\left(x_{j}-E\left(x_{j}\right)\right)\right\} \\
& +w(k) .
\end{aligned}
$$

where

$$
\begin{array}{ll}
\hat{p}_{k+1}= & \begin{array}{l}
\text { estimated price for the } k+1 \text { th trading period } \\
E\left(p_{k+1} \mid k\right) \\
p_{k}
\end{array} \\
\begin{array}{l}
\text { (e.g., day-ahead energy prices); } \\
\text { energy price of the } k \text { th trading period (e.g., } \\
\text { day-ahead energy prices); } \\
j \text { th regression used for price estimation (e.g., } \\
\text { price-elasticity); } \\
\text { price model parameters to be estimated, for }
\end{array} \\
\begin{array}{l}
j=1, \ldots, N ; \\
A_{j}
\end{array} \\
\operatorname{cov}\left(p_{k}, x_{j}\right) \quad \begin{array}{l}
\text { covariance between the } k \text { th price and } j \text { th re- } \\
\text { gression; }
\end{array} \\
\begin{array}{l}
\text { variance of the } j \text { th regression; } \\
\operatorname{var}\left(x_{j}\right)
\end{array} \\
w(k) & \begin{array}{l}
\text { process noise (normally distributed with zero } \\
\text { mean and unity variance). }
\end{array}
\end{array}
$$


The mean square error function to be minimized in the leastsquare estimation (LSE) method is as follows:

$$
\begin{aligned}
e_{k+1}= & \operatorname{var}\left(\varepsilon\left(p_{k+1} \mid x_{j}\right)\right) \\
= & \operatorname{var}\left(p_{k+1}\right) \\
& -\sum_{j=1}^{N}\left\{A_{j} \operatorname{cov}\left(p_{k}, x_{j}\right) \operatorname{var}\left(x_{j}\right)^{-1} \operatorname{cov}\left(x_{j}, p_{k}\right)\right\}
\end{aligned}
$$

The strategic behavior (discrete supply function) of the power suppliers in the market at time step $\mathrm{k}$ is a function of demand elasticity, system residual demand, transmission reliability index, generation reliability index, fuel cost, and market clearing price, which by nature, are random variables. If energy market participants behave rationally, they will follow the energy market operator rules to hedge against price volatility and loss of profit risk. For example, they will try to bid/offer within ten percent tolerance of the last trading period's clearing price in order to win in the market [11]. Mathematical modeling of the players' strategic bidding practices is currently an active area of research [9], [10].

As was mentioned earlier, the prediction of the value of the interrupted load by a power marketer (such as a major power utility with numerous customers at different zones) is necessary in order to design optimal pricing tariffs and pricing of spinning reserve. The value of lost load (VOLL) is the amount of money that a customer is willing to pay at the time step $\mathrm{k}$ for not being interrupted. The values of VOLL are different among zones because:

1) Due to transmission capacity constraints (congestion), the reliability must run (RMR) and spinning reserve (SR) requirements vary among zones;

2) Different zones have different loads. This will increase the amount of RMR and SR requirements;

3) The spinning reserve price has two components: (1) capacity price, and (2) execution price. The following formulation is applied to calculate zonal VOLL:

$$
\begin{aligned}
\mathrm{SRP}_{k} & =r_{k}+\mathrm{LOLI}_{k} \cdot p_{k} \\
\operatorname{VOLL}_{k} & =\mathrm{SRP}_{k} \cdot \mathrm{SR}_{k} .
\end{aligned}
$$

where
$\mathrm{SRP}_{k}$
predicted spinning reserve price at hour $k$, in $\mathrm{SR}_{k}$ U.S. dollars per megawatt hour;
$\mathrm{SR}_{k} \quad$ day-ahead spinning reserve requirement at hour $k$, in megawatt hours;
$r_{k} \quad$ predicted capacity price for SR at hour $k$, in U.S. dollars per megawatt hour;
$\mathrm{LOLI}_{k}=$ loss of load index at hour $k$;
$\mathrm{SR}_{k} / D_{k}$
$p_{k}$
$D_{k}$ predicted energy price at hour $k$, in U.S. dollars per megawatt hour;
expected demand for trading period $k$, in megawatt hour.

The same methodology can be used for day-ahead SR capacity price prediction.
Based on the above formulation, the MMB can determine the market value of the load to be dropped and the price of the spinning reserve. Now, the load response program can be designed and the state reserve requirements can be determined. In Section III, the leader-follower optimization will be described to compute the MCFs that the players need to satisfy in their bids in the auction market. It is recognized that the time dependent system reliability index is a major factor in the proposed formulation. Since this index has a major impact on the offering price of market players and will determine the reliability index of the system, it is expected that both major players and the state power authority would be interested in collecting the data to compute this index. The real time data collection capabilities that are available today can be used to record outages of transmission lines, generators and transformers, including the trading behaviors of the players. The author agrees that a major research effort is needed in this area. It is also expected that major power market players are collecting data to estimate some forms of this index when determining their offering price.

\section{LEADER-FOLLOWER OPTIMIZATION}

We will use the well-established Stackelberg leader-follower game theory [8], [19]-[21]. In these types of problems, there are a number of decision makers: the leader who declares the constraints of participating in the leader-proposed market opportunities and followers who are interested in the market, but must satisfy the leader's constraints. We will designate the leader as the MMB with the decision control variable vector $x$, and the followers as market players with decision control variable $y$. The constraints are defined as MCF. The market players are optimizers who are interested in maximizing their payoff function by knowing MCF. That is for some $x=Y$, the leader objectives are satisfied.

Let us designate the decision control variables for player " $i$ " as $y_{i}^{p}(k)$ in megawatts for the quantity of power with superscript " $p$ " and $y_{i}^{\lambda p}$ in U.S. dollars per megawatt hour to be the price offered to the auction market at a bus " $n$." Let us designate $x_{i}^{p}(k)$ in megawatts for the quantity of power with superscript " $p$ " and $x_{i}^{\lambda p}(k)$ in dollars per megawatt hour to be the price under the MMB controls at bus " $n$." Based on the above discussion, the MMB convex objective function can be defined as

$$
\begin{aligned}
G_{n}\left(y_{i}^{p}, x_{i}^{p}, x_{i}^{\lambda p}, y_{i}^{\lambda p}\right)= & \sum_{i=1}^{m n}\left(\lambda_{i}^{p}(k) \lambda_{i}^{\lambda p}(k)\right)^{2} \\
& +\sum_{i=1}^{m n}\left(x_{i}^{p} x_{i}^{\lambda p}(k)\right)^{2} \\
n= & 1,2, \ldots N .
\end{aligned}
$$

The above equation describes the cost of the system operation at bus " $n$ " from the perspective of the MMB in U.S. dollars per hour. Note that since the MMB has control over the load and/or system reserve, the MMB is also a player in the market, and it can influence the market players' decision to make a rational offer at bus " $n$." Note also

$$
\begin{aligned}
& y_{i}(k)=y_{i}^{p}(k) y_{i}^{\lambda p}(k) \\
& x_{i}(k)=x_{i}^{p}(k) x_{i}^{\lambda p}(k)
\end{aligned}
$$


where $y_{i}(k)$ is in U.S. dollars per hour price offer for generator " $i$ " and $x_{i}(k)$ is U.S. dollars per hour for the cost of load response curtailment or the reserve provided by the MMB. The MMB recognizes that for the MCFs to result in an acceptable schedule, they must be computed subject to system operating constraints such as power flow constraints. Let us consider the simple constraint that the MMB will be interested in as expressed by the scheduled power injections at each system bus " $n "$.

$$
C_{n}\left(y_{i}^{p}, x_{i}^{p}\right)=\sum_{i=1}^{m n}\left(y_{i}^{p}(k)-x_{i}^{p}(k)\right)^{2} \quad n=1,2, \ldots, N .
$$

The MMB seeks to provide system security as dedicated by power flow constraints as described by (6) without creating congestion in the transmission systems, and " $m n$ " is the number of participating generators at bus " $n$." Note that $x_{i}^{p}(k)$ is an algebraic quantity with a positive sign for generation and a negative sign for the loads.

The MMB will perform the optimum power flow (OPF) and determine the rational price offer and the amount of the schedule for each participating generator " $i$ " at bus " $n$ " as target values to formulate the MCF functions to be described next. Let us designate $X_{i}(k)$ in U.S. dollars per hour by the MMB as its target cost for the generator type $i$. Let us also designate $Y_{i}^{p}(k)$ and $Y_{i}^{\lambda p}(k)$ as the desired MMB for the active power and price to be selected by the players. From the MMB's perspective, the optimal operation of the system can be achieved if the market players select the $Y_{i}^{p}(k)$ and $Y_{i}^{\lambda p}(k)$ such that the hourly cost of operation of generator " $i$ " is equal to $X_{i}(k)$. To induce the players to select the MMB targets, the MMB defines the MCF's as follows:

$$
\begin{aligned}
x_{i}(k)=X_{i}(k)+\alpha_{i}(k)\left(y_{i}^{p}(k)-Y_{i}^{p}(k)\right) & \\
& +\beta_{i}(k)\left(y_{i}^{\lambda p}(k)-Y_{i}^{\lambda p}(k)\right)
\end{aligned}
$$

The computation of $\alpha_{i}$ and $\beta_{i}$ are given in [8].

For example, for a three-bus system, we will have the following set of equations:

$$
\begin{aligned}
& \alpha_{1}(k) y_{1}^{p}(k)+\beta_{1}(k) y_{1}^{\lambda p}(k)-\alpha_{1} Y_{1}^{p}(k)-\beta_{1} Y_{1}^{\lambda p}(k)=0 \\
& \alpha_{2}(k) y_{2}^{p}(k)+\beta_{2}(k) y_{2}^{\lambda p}(k)-\alpha_{2} Y_{2}^{p}(k)-\beta_{2} Y_{2}^{\lambda p}(k)=0 \\
& \alpha_{3}(k) y_{3}^{p}(k)+\beta_{3}(k) y_{2}^{\lambda p}(k)-\alpha_{2} Y_{2}^{p}(k)-\beta_{3} Y_{2}^{\lambda p}(k)=0
\end{aligned}
$$

The market players would have to satisfy the above MCF functions in developing the generation quantity and price bids at every bus " $n$ " for each generator " $i$." Note that each type of generator " $i$ " with the same incremental cost will have the same type of MCF functions. The MCFs are developed to satisfy the system's security and reliability requirement, and the offer bids will not result in congestion since the players will be induced to select the MMB targets to maximize their profits. Since the MMB is estimating the players' cost and may set its target values too high, the MCFs described by (7) constitute a set of constraints that should be set less than zero. To satisfy these constraints, the players are free to select their price offered and the amount of energy offered to satisfy the MCF functions. However, to win a higher share of the market, they would need to submit competitive bids. To ensure that the players will not withhold power from the forward market, the time horizon of operation must to be in order of a few years so that new players will be induced to build new plants where needed and compete in the market. In addition, since the MMB is also a player in the market, he or she can also compete with other players to supply the loads. The energy supply of the MMB reserve will come from several sources such as the load response program and the state reserve that has been acquired for the time horizon of planned operation. This indicates that MCF functions are dynamic in nature and are valid for a given anticipated load, generation, and transmission system. The players know that the MMB is not bluffing and will release the reserve when the market could be subjected to gaming because of the news of loss of a large unit. For the long-term forward market of a thousand MW unit, the MMB will study the system and compute the MCF's, then send them to the market. Players are ensured that by satisfying the MCF functions, their planned generation will be coordinated with the transmission systems, and they are properly compensated in the return on their investment. The main objective of the MMB is planned operation. The MMB seeks to minimize the amount of power that must be purchased in the spot market, where the price could be high due to any sudden contrived or real outages. In fact, the MMB, through the state power reserve, will control the spinning reserve requirements and load response program to effectively control the price of the spot market. It should be apparent that since the MMB determines the target values for operation of the systems in the forward market, the MMB will plan the system and not the market forces that may result in congestion and blocking units under contracts in the systems. In this structure, all bids into the auction market will have a registration tag to identify the source of generation and the contract, and can be traded as a commodity after the contract is certified by the MMB.

\section{SUMmARY OF THE AlgORITHM}

Step 1) The MMB will estimate the production cost of each player based on the registered information about their unit such as fuel type, dynamic response, location of the unit, and historical data.

Step 2) The MMB will forecast the load demands for each load center.

Step 3) The MMB will determine the system reliability index, SRI, and compute the value of load loss and the expected market value of spinning.

Step 4) The MMB will develop load response programs through incentive-designed tariffs to control the reserve requirements.

Step 5) The MMB will perform an optimal power flow study and determine the optimal schedules for each participating generating unit.

Step 6) The results of the optimal power flow (OPF) study will determine the expected production output for each generator type " $i$." The OPF will determine the 
desired quantity and price TARGETS for each participating generating " $i$ " at each bus in the system.

Step 7) The MMB will formulate the MMB cost function, the system load flow constraints, and MCF functions. Then, it will formulate the global leader-follower optimization problem to compute the coefficients of MCF's in terms of the MMB targets that are defined by the system security constraints.

Step 8) The MMB will send MCFs to market players for them to satisfy when submitting acceptable bids.

Since the historical data needed for proposed algorithms are not available, a meaningful simulation cannot be performed. However, the above steps show that the MMB can monitor the market and mesh the interests of players with system reliability and security requirements. Since the reliability data profoundly affects the price of the spinning reserve, market players may consider this data proprietary. However, the MMB can collect the reliability information and use it for system security and control of energy services as outlined above.

\section{CONCLUSION}

In this paper, we have presented a framework and a solution methodology to control energy services within the restructured electricity markets. This paper takes the position that planned operation of the system is essential to ensure stable prices. Without planned operation, the electric energy system will be subjected to a cycle of deficiencies in energy production that will eventually lead to a cycle of bottlenecks and excessive generation. This cycle will repeat itself, if it is left to market forces alone to plan the operation of the system. This will result in substantial dislocation in the economy.

\section{ACKNOWLEDGMENT}

The author wishes to thank the contribution of Dr. D. Shirmohammadi of PA Consulting Group for the idea of a market monitoring structure.

\section{REFERENCES}

[1] P. J. Werbos, "Maximizing long-term gas industry profits in two minutes in lotus using neural network methods," IEEE Trans. Syst., Man, Cybern, vol. 19, pp. 315-333, Mar./Apr. 1989

[2] M. Amin, "Toward self-healing energy infrastructure systems," IEEE Comput Applicat. Power, vol. 14, pp. 20-28, Jan. 2001

[3] M. Illic, "A presentation on research challenges in the electric power systems," NSF, Apr. 1999, to be published.

[4] R. J. Thomas, "Introduction to the minitrack on restructuring the electric power industry: Emerging issues, methods and tools," in Proc. 33rd Annu. Hawaii Int. Conf. Syst. Sci., 2000, p. 90.

[5] S. Talukdar and E. Camponogara, "Collaborative nets," in Proc. 33rd Annu Hawaii Int. Conf. Syst. Sci., 2000, p. 103.

[6] F. L. Alvarado, J. Meng, W. S. Mota, and C. L. DerMarco, "Dynamic coupling between power markets and power systems," in Proc. IEEE Power Eng. Soc. Summer Meeting, vol. 4, 2000, pp. 2201-2205.

[7] N. S. Rau, "Assignment of capability obligation to entities in competitive markets-the concept of reliability equity," IEEE Trans. Power Syst., vol. 14, pp. 884-889, Aug. 1999.

[8] M. Shahidehpour and M. Alomoush, Elements of Restructured Electric Power Systems. New York: Marcel Dekker, 2001.
[9] J. W. Lamont and S. Rajan, "Strategic bidding in an energy brokerage," IEEE Trans. Power Syst., vol. 12, pp. 1729-1733, Nov. 1997.

[10] G. Gross, D. J. Finlay, and G. Deltas, "Strategic bidding in electricity generation supply markets," in Proc. IEEE Power Eng. Soc. Annu. Conf. Proc., vol. 1, New York, NY, Feb. 1999, pp. 309-315.

[11] P. Skantze and J. Chapman, "Price dynamics in the deregulated california energy market," in IEEE Power Eng. Soc. Annu. Conf. Proc., vol. 1, New York, NY, Feb. 1999, pp. 287-293.

[12] W. W. Hogan, "A market power model with strategic interaction in electricity networks," The Energy J., vol. 18, no. 4, pp. 107-141.

[13] D. Shirmohammadi, B. Wollenberg, A. Vojdani, P. Sandrin, M. Pereira, F. Rahimi, T. Schneider, and B. Scott, "Transmission dispatch and congestion management in the emerging energy market structures," IEEE Trans. Power Syst., vol. 13, pp. 1466-1474, Nov. 1998.

[14] R. Billinton and R. Kavki, "Capacity reserve assessment using system well-being analysis," IEEE Trans. Power Syst., vol. 14, pp. 433-438, May 1999.

[15] S. Dekrajangpetch, G. B. Sheble, and A. J. Conejo, "Auction implementation problems using Lagrangian relaxation," IEEE Trans. Power Syst. vol. 14, pp. 82-88, Feb. 1999.

[16] J. Mitra and C. Singh, "Capacity assistance distributions for arbitrarily configured multi-area networks," IEEE Trans. Power Syst., vol. 12, pp. 1530-1535, Nov. 1997.

[17] B. H. Kim and M. L. Baughman, "The economic efficiency impacts of alternatives for revenue reconciliation," IEEE Trans. Power Syst., vol. 12, pp. 1129-1135, Aug. 1997.

[18] J. A. Casazza, "Electricity choice: Pick your poison," Public Utility Fortnightly, vol. 139, no. 5, pp. 42-49, Mar. 2001.

[19] A. Keyhani, J. Cruz Jr., and M. A. Simaan, "Market monitoring and leader follower incentive control of ancillary services," in Proc. 32nd Annu. Hawaii Int. Conf. Syst. Sci., Jan. 4, 1999, p. 144.

[20] T. Basar and J. B. Cruz Jr., "Deterministic incentives," in Systems \& Control Encyclopedia Theory, Technology, Applications, M. G. Singh, Ed. Oxford, New York: Pergamon Press, 1987, vol. 2, pp. 993-995.

[21] T. Basar and H. Selbuz, "Closed-loop stackelberg strategies with applications in the optimal control of multilevel systems," IEEE Trans. Automat. Control, vol. AC-24, pp. 166-179, Apr. 1979

[22] M. Simaan and J. B. Cruz Jr., "On the stackelberg strategy in nonzero-sum games," J. Optim. Theory Applicat., vol. 11, pp. 533-555, May 1973.

[23] Y. K. Kwok, Mathematical Models of Financial Derivatives. New York: Springer-Verlag, 1998

[24] C. Gourieroux and A. Monfort, Time Series and Dynamic Models. Cambridge, U.K.: Cambridge Univ. Press, 1997.

[25] J. Keppo and M. Rasanen, "Pricing of electricity tariffs in competitive markets," Elsevier Sci. Inc., J. Energy Econ., vol. 21, pp. 213-223, 1999.

[26] D. W. Bunn, "Forecasting loads and prices in competitive power markets," Proc. IEEE, pp. 163-169, Feb. 2000.

[27] A. Kian and A. Keyhani, "Stochastic price modeling of electricity in deregulated energy markets," in Proc. Hawaii Int. Conf. Syst. Sci., Jan. 4-7, 2000.

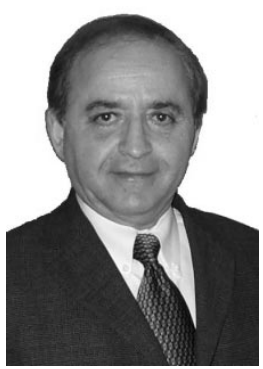

Ali Keyhani (F'98) research interests include the areas of electromechanical systems, power systems, control and operation, leader-follower optimization of restructured energy systems, power electronics, design of electric machines, and parameter estimation.

Dr. Keyhani is the past Chairman of the Electric Machinery Committee and the past Editor of IEEE TRANSACTIONS On ENERGY CONVERSION. He has authored many papers in the IEEE Transactions journals on control of power systems, machine modeling, parameter estimation, power electronic systems, and design of virtual test beds for variable speed drive

systems.

Dr. Keyhani is a recipient of The Ohio State University's College of Engineering Research Award for 1989 and 1999. 